\title{
Effects of metformin on survival outcomes of pancreatic cancer patients with diabetes: A meta-analysis
}

\author{
WENXIU XIN ${ }^{1,2^{*}}$, LUO FANG ${ }^{3}$, QILU FANG ${ }^{1}$, XIAOWEI ZHENG ${ }^{1}$ and PING HUANG ${ }^{1,2 *}$ \\ ${ }^{1}$ Laboratory of Clinical Pharmacy, Zhejiang Cancer Hospital; ${ }^{2}$ Key Laboratory of Head and Neck Cancer Translational \\ Research of Zhejiang Province; ${ }^{3}$ Zhejiang Key Laboratory of Diagnosis and Treatment Technology on Thoracic \\ Oncology (Lung and Esophagus), Zhejiang Cancer Hospital, Hangzhou, Zhejiang 310022, P.R. China
}

Received August 2, 2017; Accepted December 6, 2017

DOI: $10.3892 / \operatorname{mco} .2017 .1541$

\begin{abstract}
Pancreatic cancer risk is reduced by metformin treatment in patients with diabetes. However, the effect of metformin on pancreatic cancer overall survival is unclear. The aim of the present study was to determine the association between metformin and clinical outcomes of pancreatic cancer patients with diabetes. An electronic and manual search was conducted using PubMed, Web of Science, Medline-Ovid and Cochrane Library databases between the beginning and March 31, 2017. A total of 8 studies consisting of 4,293 patients with pancreatic cancer with diabetes were included, comprising 2,033 patients who had received metformin and 2,260 patients who had not. The meta-analysis showed that metformin was associated with a relative survival benefit in pancreatic cancer patients [hazard ratio (HR), 0.81; 95\% confidence interval (CI), 0.70-0.93]. These associations were also observed in subgroups of Asian countries 0.64 (95\% CI, 0.52-0.80) and Western countries 0.88 (95\% CI, 0.82-0.95), as well as diabetes (no indication of diabetes type). Excluding the studies considered as be prone to immortal time bias resulted in HRs (95\% CIs) of $0.86(0.69-1.07)$. The results of this study support the notion that the use of metformin may improve the overall survival of patients with pancreatic cancer with concurrent diabetes. However, the proposed beneficial effect of metformin on pancreatic cancer survival may be based on immortal time bias. Further carefully designed studies with high quality are warranted to confirm this efficacy.
\end{abstract}

Correspondence to: Dr Ping Huang, Laboratory of Clinical Pharmacy, Zhejiang Cancer Hospital, 1 Guangji Road, Hangzhou, Zhejiang 310022, P.R. China

E-mail: huangping1841@zjcc.org.cn

${ }^{*}$ Contributed equally

Key words: metformin, pancreatic cancer, survival, diabetes, meta-analysis

\section{Introduction}

Pancreatic cancer is the fourth leading cause of cancer-related deaths in both men and women in Western countries $(1,2)$. Despite the recent advances in surgical techniques and adjuvant therapies, prognosis for patients with pancreatic cancer remains poor. The 5-year survival rate is only $24 \%$ even in patients with early disease and margin-negative resection. For patients with pancreatic cancer present with unresectable disease, the 5-year survival rate is worse at just 2\% (3). More effective treatment strategies are urgently needed for the management of pancreatic cancer.

Metformin, a medication in the biguanide class, is used as an oral glucose-lowing agent in the treatment of type 2 diabetes mellitus (T2DM). It has been reported that metformin treatment reduces hepatic gluconeogenesis by inhibiting mitochondrial glycerophosphate dehydrogenase (4). Metformin were reported to play a potential anticancer effect through molecular mechanisms of the mammalian target of rapamycin (mTOR)-signaling pathway and ATM/LKB1/AMPK axis (5-7). Preclinical work has also added to the evidence that metformin have antineoplastic activity in pancreatic cancer cell lines (8). Considering all the results above, it is natural to regard metformin as a well-tolerated and promising agent for prevention and treatment of pancreatic cancer.

So far, there is a growing interest in investigating the role of metformin for its anticancer effect in different cancer types. Three systematic reviews assessed the effects of metformin on clinical outcomes of any type of cancer and reported that metformin was associated with a reduction in overall mortality and cancer-specific mortality (9-11). However, the results vary in the associations between different cancer types and mortality risk with metformin exposure. For example, results of several systematic reviews suggest that treatment with metformin is associated with reduced cancer mortality compared with other glucose-lowering therapies in colorectal (12), breast (13), ovarian and endometrial cancer (14), while was not associated with the reduction of mortality in prostate cancer (15).

Also, several recent observational studies have explored the association between use of metformin and clinical outcomes of pancreatic cancer. Lee et al (16) supported that metformin 
exposure was associated with improved clinical outcomes of pancreatic cancer patients. However, Hwang et al (17), Reni et al (18) and Kordes et al (19) found that metformin exposure was not associated with survival benefit in subjects with advanced pancreatic cancer. Based on these studies, the relationship between metformin use and the prognosis of pancreatic cancer in diabetic patients is still controversial. Therefore, we performed a systemic review and meta-analysis to assess the effect of metformin usage on survival outcome of patients with concurrent diabetes and pancreatic cancer.

\section{Data collection methods}

The meta-analysis was conducted in accordance with the PRISMA guidelines (19), STROBE Statement (20) as well as Cochrane Collaboration guidelines (21).

Search strategy. We searched the PubMed, Web of Science, Medline-Ovid, and Cochrane Library databases for relevant studies up to 31th March 2017, which was performed by two study investigators independently. The keywords combined with corresponding Mesh terms used for searching included (metformin or biguanide or dimethylbiguanidine) and (neoplasms or tumor or cancer or carcinoma or malignancy) and (pancreas or pancreatic). In addition, references cited in the identified studies, recent review articles, meta-analyses and other relevant studies were also scrutinized to identify potentially pertinent articles which possibly missed in the original search.

Eligibility criteria. Inclusion criteria were: (1) patients with a pathologically confirmed diagnosis of pancreatic adenocarcinoma; (2) original articles reported time to event data [hazard ratios (HRs) with $95 \%$ confidence interval (CI)] belong to association between metformin use and survival of pancreatic cancer, and (3) study design: Randomized controlled trials, cohort studies, or case-control studies. Additionally, considering that diagnosis of diabetes was regarded as an important confounder of the relationship between metformin exposure and prognosis of pancreatic cancer, we restricted this meta-analysis to studies that included only pancreatic patients pre-existing with diabetes based on medical or pathology reports. We excluded small sample size studies with no time to event data provided or low study quality. No language restriction was performed. When more than one publication reported on the same study, only the publication with most complete dataset or reported recently was included.

Data extraction. Data extraction was performed in duplicate by two independent reviewers based on the inclusion criteria listed above. Any disagreements were reconciled through group discussion. The following information was extracted from eligible articles: Publication data (study title, the first author's last name, study country), study design (clinic-based or population-based cohort studies, RCT or case-control studies), data source, cancer stage, cancer subtypes, sample size, length of follow-up, outcomes, risk estimates with their corresponding CIs, the matching variables in the multivariable model, financial disclosure documentation, and industry sponsorship.
Quality assessment. To ascertain the validity of the eligible studies, the quality of nonrandomized observational studies was evaluated in reference to the Newcastle Ottawa Scale (NOS) by two investigators (22). In this 'star system', included studies were judged on three aspects: Selection of study groups, comparability of studies groups, and the ascertainment of exposure or outcome. Based on this tool, the quality of observational studies, with nine stars at most, was categorized as low quality (less than 4 points), medium quality (a score of 5 or 6 ), or high quality (a score of 7 or higher).

Statistical analysis. Pooled HRs with 95\% CI was analyzed. Heterogeneity across included studies was analyzed by $I^{2}$ statistics and $Q$ test (23). $I^{2}$ values of $>50 \%$ or $Q$ test of $\mathrm{P}$-values less than 0.01 represented significant heterogeneity. Publication bias for observational studies was evaluated using Begg's funnel plot and Egger's test $(\mathrm{P}<0.05$ indicated the presence of publication bias) (24-26). A DerSimonian-Laird (D-L) random-effect model (27) was selected to calculate the pooled HRs for overall survival (OS). Otherwise, an inverse-variance fixed-effect meta-analysis model (28) was chosen. The subgroup analyses stratified by the potentially important factors, such as study region, diabetes type, cancer stage as well as immortal time bias, were further carried out to examine the source of possible heterogeneity. Forest plot were distinguished according to the author's surname and year of publication to illuminate the HRs with 95\% CI. All main statistical analyses were conducted using Review Manager Version 5.3 software package (Oxford, United Kingdom), while publication bias and sensitivity analysis were performed using Stata software (Stata Corp, College Station, TX, USA).

\section{Results}

Literature search. We retrieved a total of 2,121 citations through electronic and manual search. After excluding 315 duplicate and 1,778 irrelevant articles based on titles or abstracts, we finally included 28 citations seemed to meet the inclusion criteria for detailed evaluation. After reading the full text, 20 were excluded because article overlapping, no survival information, not diabetic patients and no sufficient data. At last, 8 full articles $(16,17,29-34)$ matched our inclusion criteria and included in this meta-analysis. The process of study selection is shown in a flow diagram (Fig. 1).

Characteristics of included studies and study quality. Data on first author, publication year, country, study design, study period, population, age, cancer treatment, adjusting variables and follow-up time are presented in Table I. The eight observational studies in the meta-analysis of overall survival included 4,293 pancreatic cancer patients with diabetes, including 2,033 patients who took metformin and 2,260 who did not. These studies were all published in recent years (2012 to 2017), and six studies were published in 2016. Five studies were conducted in the United States (29,31-34), two in Korea $(16,30)$, one in UK (17). These eight eligible papers are all retrospective cohort studies. Sample sizes ranged from 44 to 1916 patients. The percentage of metformin users in pancreatic cancer patients ranged from 11 to $57 \%$. Four studies $(17,31,34,35)$ explicitly mentioned exclusion of patients 


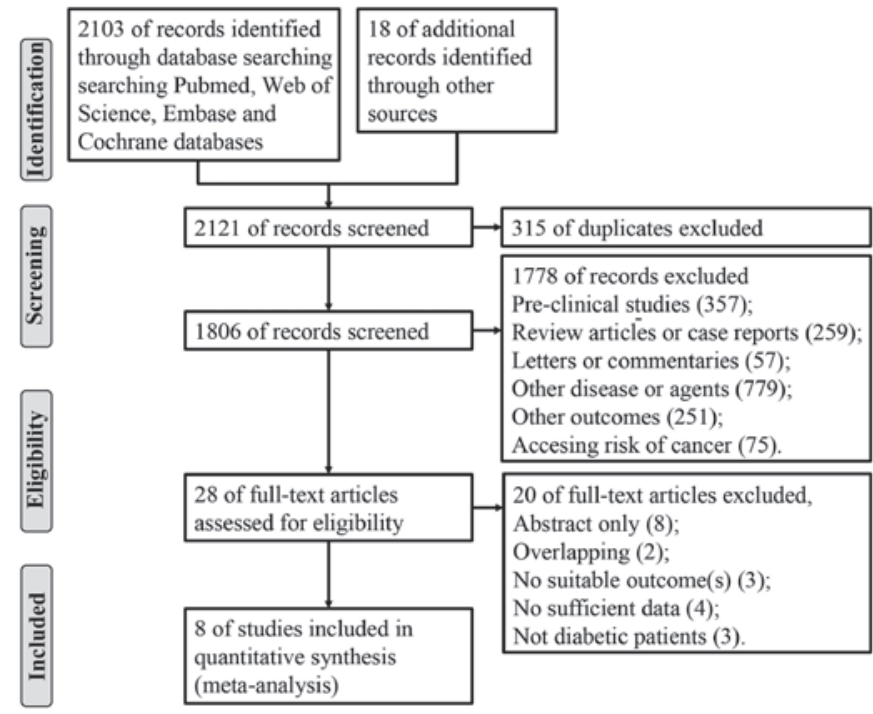

Figure 1. Flow diagram for study selection.

with type 1 diabetes mellitus. In all the 8 studies, all estimates were adjusted for potential confounders using multivariate analysis, though the variables involved were not exactly the same among them. The quality of the 8 included studies was appraised according to the NOS, and NOS scores for each study are shown in Table I. All the 8 included studies are cohort studies. Of the 8 included studies, 3 studies scored 8 and 5 studies scored 7, which showed a high quality of all the included studies.

Quantitative synthesis. The study-specific and pooled HRs for OS associated with metformin in pancreatic cancer patients are shown in Fig. 2. All the eligible studies reported the HR for OS when compared the overall survival of metformin with non-metformin use groups. In a total of 8 studies, 4 studies reported a statistically decreased risk of death from all causes in pancreatic cancer patients with diabetes by multivariate analysis $(16,30,32,33)$. However, no statistically significant differences were observed in the other 4 studies $(17,29,31,34)$. Considering the presence of significant heterogeneity among all the included studies $\left(\mathrm{P}=0.01 ; I^{2}=64 \%\right)$, we used the random-effects models to conduct the pooled analysis. The pooled results demonstrated that metformin administration to patients with pancreatic cancer and diabetes was associated with a $19 \%$ reduced risk for overall mortality compared with those who did not receive metformin (HR: 0.81; 95\% CI: $0.70-0.93$ by random effect).

In a fixed model analysis stratified by study region (Asian or Western countries), we found that metformin exposure was associated with a significantly reduced risk for death in Asian countries (HR: 0.64; 95\% CI: $0.52-0.80$ by fixed effect; $\mathrm{P}=0.57$ for heterogeneity; $I^{2}=0 \%$ ). In the subgroup of Western countries, the meta-analysis demonstrated that the HR of OS was 0.88 (95\% CI: $0.82-0.95$ by fixed effect; $\mathrm{P}=0.08$ for heterogeneity; $I^{2}=50 \%$ ) (Table II). We next performed subgroup analyses by diabetes type (diabetes or T2DM). In the subgroup of patients with diabetes (no indication of diabetes type), metformin was still associated with reduced death risk (HR: 0.85 ; 95\% CI: $0.78-0.92$ by fixed

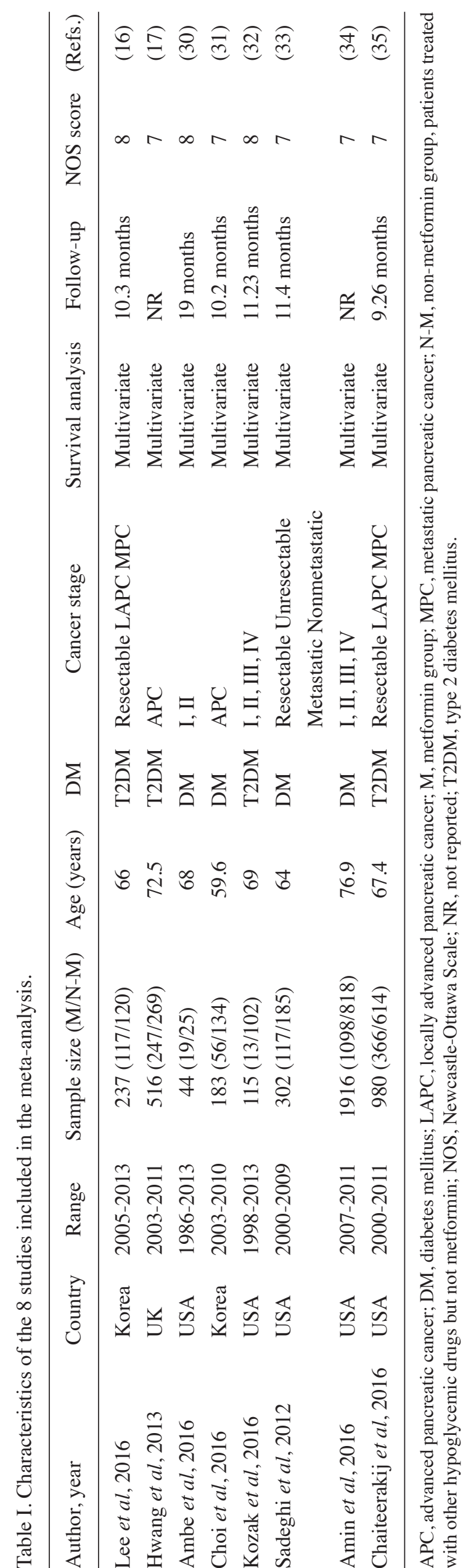


Table II. Associations between metformin and overall survival.

\begin{tabular}{|c|c|c|c|c|c|}
\hline \multirow[b]{2}{*}{ Criteria } & \multirow[b]{2}{*}{$\mathrm{N}$} & \multicolumn{2}{|c|}{ Pooled HR } & \multicolumn{2}{|c|}{ Heterogeneity } \\
\hline & & Fixed $(95 \%$ CI) & Random (95\% CI) & $I^{2}(\%)$ & P-value \\
\hline Main effect & 8 & $0.86[0.80,0.92]$ & $0.81[0.70,0.93]$ & 60 & 0.01 \\
\hline \multicolumn{6}{|l|}{ Region } \\
\hline Asian country & 2 & $0.64[0.52,0.80]$ & $0.64[0.52,0.80]$ & 0 & 0.57 \\
\hline Western country & 6 & $0.88[0.82,0.95]$ & $0.87[0.76,1.00]$ & 50 & 0.08 \\
\hline \multicolumn{6}{|l|}{ Diabetes type } \\
\hline Diabetes & 4 & $0.85[0.78,0.92]$ & $0.76[0.62,0.94]$ & 50 & 0.11 \\
\hline $\mathrm{T} 2 \mathrm{DM}$ & 4 & $0.88[0.78,0.99]$ & $0.84[0.64,1.10]$ & 73 & 0.01 \\
\hline \multicolumn{6}{|l|}{ Cancer stage } \\
\hline Early & 1 & $0.54[0.16,1.86]$ & - & - & - \\
\hline Advances & 2 & $0.97[0.81,1.17]$ & $0.89[0.56,1.42]$ & 81 & 0.02 \\
\hline All stage & 5 & $0.84[0.78,0.90]$ & $0.78[0.67,0.91]$ & 60 & 0.04 \\
\hline \multicolumn{6}{|l|}{ Immortal time bias } \\
\hline With & 4 & $0.79[0.69,0.89]$ & $0.73[0.59,0.92]$ & 49 & 0.12 \\
\hline Without & 4 & $0.88[0.82,0.96]$ & $0.86[0.69,1.07]$ & 68 & 0.03 \\
\hline
\end{tabular}

CI, confidence interval; HRs, Hazard ratios; N, number. of studies; T2DM, type 2 diabetes mellitus.

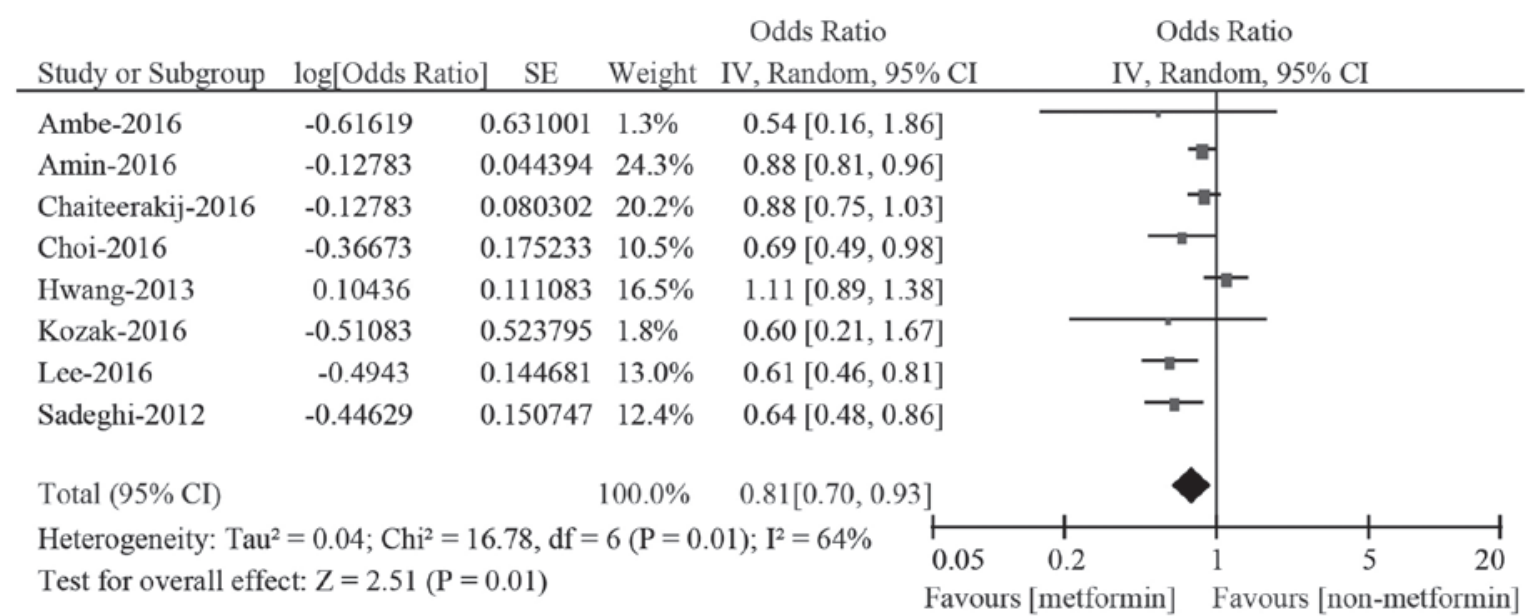

Figure 2. HRs for overall survival associated with metformin exposure vs. non-use. HRs, hazard ratios; CI, confidence interval; df, degrees of freedom.

effect; $\mathrm{P}=0.11$ for heterogeneity; $I^{2}=50 \%$ ). In the subgroup of patients with T2DM, the relative survival benefit associated with metformin reversed (HR: $0.84 ; 95 \%$ CI: $0.64-1.10$ by random effect; $\mathrm{P}=0.01$ for heterogeneity; $I^{2}=73 \%$ ) (Table II). Analyses on cancer stage did not show beneficial associations besides overall survival among advanced pancreatic cancer patients (HR: 0.89 ; 95\% CI: $0.56-1.42$ by random effect; $\mathrm{P}=0.02$ for heterogeneity; $I^{2}=81 \%$ ). Details of exposure assessment were not presented in studies by Ambe et al (30), Chaiteerakij et al (35) and Choi et al (31), and metformin use with more than 1 month after cancer diagnosis in the study by Lee et al (16), perhaps these studies were prone to immortal time bias. Excluding the studies considered as be prone to immortal time bias resulted in HRs (95\% CIs) of $0.86(0.69-1.07)$.

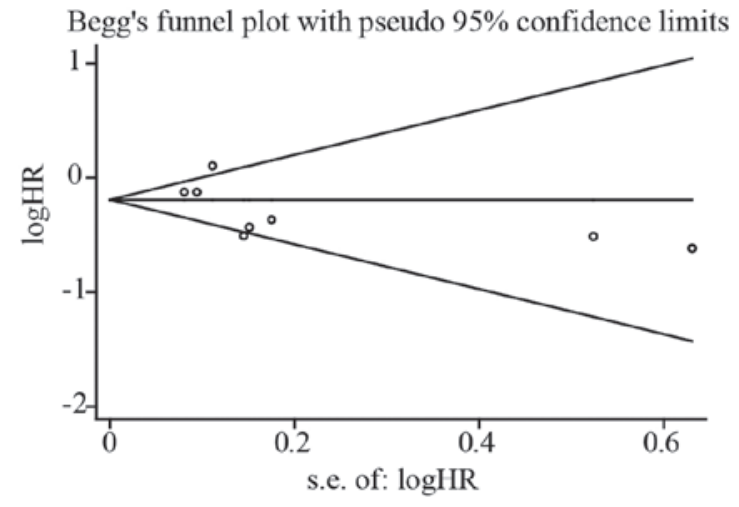

Figure 3. Begg's funnel plot of the included studies for publication bias test on the association of metformin exposure with survival outcomes of pancreatic cancer patients with diabetes. 
Sensitivity analysis and publication bias. Taking into account the large variations in the covariates of the included studies, we conducted a sensitivity analysis. Sensitivity analysis was performed by sequential omission of any individual studies to investigate the influence of single data set on the overall meta-analysis. Sensitivity analysis indicated that removal of each study from the meta-analysis did not overthrow the result of the present pooled analysis. However, results in heterogeneity was reduced when remove the study by Hwang et al (17). Presence of publication bias evaluated using Begg's funnel plot $(\mathrm{P}=0.458$ for metformin on $\mathrm{OS})$ and Egger's linear regression test $(\mathrm{P}=0.195$ for metformin on $\mathrm{OS})$ showed no obvious publication bias (Fig. 3).

\section{Discussion}

Our meta-analysis assessed the effects of metformin exposure on overall survival of pancreatic cancer patients with diabetes mellitus. In fact, our meta-analysis including 4,293 participants from eight cohort studies revealed that diabetic patients with pancreatic cancer using metformin achieved an estimated survival benefit of $19 \%$ compared with non-metformin users. In sub-group analysis, metformin administration significantly associated with a good prognosis in patients from Asian. As shown in Western, metformin exposure may also associate with a good prognosis in these patients but the effect was modest.

As a well-accepted anti-diabetes drug, the potential anti-cancer effects of metformin have not been fully elucidated. Metformin modulates several signal pathways crucial to cancer progression. It inhibits lipogenic pathways and activates AMP-activated kinase (AMPK) (36-38), an inhibitor of cellular proliferation via the mammalian target of rapamycin (mTOR) pathway $(39,40)$ to decrease cell metabolism status and reduce serum concentrations of insulin and insulin growth factor I (IGF-I) $(41,42)$. Although experimental evidences have confirmed the anti-tumor effect of metformin, results of clinical and epidemiological researches are complex and inconsistent. Our meta-analysis are inconsistent with a previous meta-analysis of overall survival in pancreatic cancer patients with concurrent diabetes, which included two observational studies and found that metformin use was associated with a survival benefit (HR: 0.668; 95\% CI: 0.397-1.125) in patients with resected pancreatic cancer, but no statistically significant difference was found (29).

A number of potential limitations need to be considered in this meta-analysis. First, the included studies are mainly retrospective cohort studies. No randomized controlled trials or prospective studies were included, which reduced the reliability of evidence. Second, high $I^{2}$ indicated high heterogeneity between studies, which were actualized in a mixture of populations with different treatment background and diverse inclusion criteria, study population, and adjustment. Third, the included studies didn't show the impact of diabetes type, data of diabetes onset, the concentration or duration of metformin exposure. Thus, the observed benefit from concentration or duration of metformin treatment cannot be clearly defined. Fourth, the impact of other hypoglycemic agents such as insulin, sulfonylureas and thiazolidinedione were only adjusted in one study (17), which might inversely affect clinical outcomes of pancreatic cancer. Finally, after excluding studies prone to have immortal time bias, meta-analysis of existing studies does not support a survival benefit (HR: $0.86,95 \%$ CI: $0.69-1.07, I^{2}=68 \%$ ), which suggest that the proposed beneficial effect of metformin on cancer survival might be based on immortal time bias.

There are also several strengths in this meta-analysis. First, one strength of this current meta-analysis was a comprehensive search strategy and inclusion criteria to extract as much information from the literature as possible, including information from any publication type and any language. Second, we performed sensitivity analysis to investigate whether any single study changed the results, and the results showed the robustness of the conclusions. Third, based on the NOS scores, all the eligible studies in the meta-analysis were of high quality with stars ranged from 7-8. At last, both qualitative analysis by Begg's test and Egger's test showed no major publication bias.

In summary, our finding suggests that metformin usage in pancreatic cancer patients with concurrent diabetes seem to have an improved survival outcome. However, the proposed beneficial effect of metformin on pancreatic cancer survival may be based on immortal time bias. Methodological challenges of pharmacoepidemiologic studies have to be taken into account in observational studies of metformin in pancreatic cancer. Further carefully designed observational studies and potentially RCTs should be designed to improve the study quality, as well as taking several confounding factors into consideration, including date of diabetes onset, intensity and duration of metformin exposure as well as other clinical characteristics.

\section{Acknowledgements}

The present study was supported by Zhejiang Medical Technology and Education (No. 2015RCB006).

\section{References}

1. Jemal A, Bray F, Center MM, Ferlay J, Ward E and Forman D: Global cancer statistics. CA Cancer J Clin 61: 69-90, 2011.

2. Tempero MA, Malafa MP, Behrman SW, Benson AB III, Casper ES, Chiorean EG, Chung V, Cohen SJ, Czito B, Engebretson A, et al: Pancreatic adenocarcinoma, version 2.2014: Featured updates to the NCCN guidelines. J Natl Compr Canc Netw 12: 1083-1093, 2014.

3. Inoki K, Zhu T and Guan KL: TSC2 mediates cellular energy response to control cell growth and survival. Cell 115: 577-590, 2003.

4. Madiraju AK, Erion DM, Rahimi Y, Zhang XM, Braddock DT, Albright RA, Prigaro BJ, Wood JL, Bhanot S, MacDonald MJ, et al: Metformin suppresses gluconeogenesis by inhibiting mitochondrial glycerophosphate dehydrogenase. Nature 510: 542-546, 2014.

5. Del Barco S, Vazquez-Martin A, Cufí S, Oliveras-Ferraros C, Bosch-Barrera J, Joven J, Martin-Castillo B and Menendez JA: Metformin: Multi-faceted protection against cancer. Oncotarget 2: 896-917, 2011.

6. Ben Sahra I, Le Marchand-Brustel Y, Tanti JF and Bost F: Metformin in cancer therapy: A new perspective for an old antidiabetic drug? Mol Cancer Ther 9: 1092-1099, 2010.

7. Jalving M, Gietema JA, Lefrandt JD, de Jong S, Reyners AK, Gans RO and de Vries EG: Metformin: Taking away the candy for cancer? Eur J Cancer 46: 2369-2380, 2010.

8. Luo Q, Hu D, Hu S, Yan M, Sun Z and Chen F: In vitro and in vivo anti-tumor effect of metformin as a novel therapeutic agent in human oral squamous cell carcinoma. BMC Cancer 12: $517,2012$. 
9. Zhang P, Li H, Tan X, Chen L and Wang S: Association of metformin use with cancer incidence and mortality: A meta-analysis. Cancer Epidemiol 37: 207-218, 2013.

10. Zhang ZJ and Li S: The prognostic value of metformin for cancer patients with concurrent diabetes: A systematic review and meta-analysis. Diabetes Obes Metab 16: 707-710, 2014.

11. Yin M, Zhou J, Gorak EJ and Quddus F: Metformin is associated with survival benefit in cancer patients with concurrent type 2 diabetes: A systematic review and meta-analysis. Oncologist 18 $1248-1255,2013$

12. Meng F, Song L and Wang W: Metformin improves overall survival of colorectal cancer patients with diabetes: A meta-analysis. J Diabetes Res 2017: 5063239, 2017.

13. Xu H, Chen K, Jia X, Tian Y, Dai Y, Li D, Xie J, Tao M and Mao Y: Metformin use is associated with better survival of breast cancer patients with diabetes: A meta-analysis. Oncologist 20: 1236-1244, 2015.

14. Tang YL, Zhu LY, Li Y, Yu J, Wang J, Zeng XX, Hu KX, Liu JY and Xu JX: Metformin use is associated with reduced incidence and improved survival of endometrial cancer: A meta-analysis. BioMed Res Int 2017: 5905384, 2017.

15. Deng D, Yang Y, Tang X, Skrip L, Qiu J, Wang Y and Zhang F: Association between metformin therapy and incidence, recurrence and mortality of prostate cancer: Evidence from a meta-analysis. Diabetes Metab Res Rev 31: 595-602, 2015.

16. Lee SH, Yoon SH, Lee HS, Chung MJ, Park JY, Park SW, Song SY, Chung JB and Bang S: Can metformin change the prognosis of pancreatic cancer? Retrospective study for pancreatic cancer patients with pre-existing diabetes mellitus type 2. Dig Liver Dis 48: 435-440, 2016.

17. Hwang AL, Haynes K, Hwang WT and Yang YX: Metformin and survival in pancreatic cancer: A retrospective cohort study. Pancreas 42: 1054-1059, 2013.

18. Reni M, Dugnani E, Cereda S, Belli C, Balzano G, Nicoletti R, Liberati D, Pasquale V, Scavini M, Maggiora P, et al: (Ir)relevance of metformin treatment in patients with metastatic pancreatic cancer: An open-label, randomized phase II trial. Clin Cancer Res 22: 1076-1085, 2016.

19. Kordes S, Pollak MN, Zwinderman AH, Mathôt RA Weterman MJ, Beeker A, Punt CJ, Richel DJ and Wilmink JW: Metformin in patients with advanced pancreatic cancer: A double-blind, randomised, placebo-controlled phase 2 trial. Lancet Oncol 16: 839-847, 2015.

20. Moher D, Liberati A, Tetzlaff J and Altman DG; PRISMA Group: Preferred reporting items for systematic reviews and meta-analyses: The PRISMA statement. Int J Surg 8: 336-341, 2010.

21. von Elm E, Altman DG, Egger M, Pocock SJ, Gøtzsche PC and Vandenbroucke JP; STROBE Initiative: The strengthening the reporting of observational studies in epidemiology (STROBE) statement: Guidelines for reporting observational studies. J Clin Epidemiol 61: 344-349, 2008.

22. Higgins JPT: GSe: Cochrane handbook for systematic reviews of interventions Version 5.1.0 (updated March 2011). The Cochrane Collaboration available from www.cochrane-handbook.org. Accessed March 2, 2016

23. Wells GA, Shea B, O'Connell D, Peterson J, Welch V, Losos M and Tugwell P: The Newcastle-Ottawa Scale (NOS) for assessing the quality of non-randomized studies in meta-analysis, 2000.

24. van Houwelingen HC, Arends LR and Stijnen T: Advanced methods in meta-analysis: Multivariate approach and meta-regression. Stat Med 21: 589-624, 2002.

25. Copas J and Shi JQ: Meta-analysis, funnel plots and sensitivity analysis. Biostatistics 1: 247-262, 2000.

26. Begg CB and Mazumdar M: Operating characteristics of a rank correlation test for publication bias. Biometrics 50: 1088-1101, 1994.
27. Egger M, Davey Smith G, Schneider M and Minder C: Bias in meta-analysis detected by a simple, graphical test. BMJ 315 : 629-634, 1997.

28. DerSimonian R and Laird N: Meta-analysis in clinical trials. Control Clin Trials 7: 177-188, 1986

29. Mantel N and Haenszel W: Statistical aspects of the analysis of data from retrospective studies of disease. J Natl Cancer Inst 22: 719-748, 1959.

30. Ambe CM, Mahipal A, Fulp J, Chen L and Malafa MP: Effect of metformin use on survival in resectable pancreatic cancer: A single-institution experience and review of the literature. PLoS One 11: e0151632, 2016

31. Choi Y, Kim TY, Oh DY, Lee KH, Han SW, Im SA, Kim TY and Bang YJ: The impact of diabetes mellitus and metformin treatment on survival of patients with advanced pancreatic cancer undergoing chemotherapy. Cancer Res Treat 48: 171-179, 2016.

32. Kozak MM, Anderson EM, von Eyben R, Pai JS, Poultsides GA, Visser BC, Norton JA, Koong AC and Chang DT: Statin and metformin use prolongs survival in patients with resectable pancreatic cancer. Pancreas 45: 64-70, 2016

33. Sadeghi N, Abbruzzese JL, Yeung SC, Hassan M and Li D: Metformin use is associated with better survival of diabetic patients with pancreatic cancer. Clin Cancer Res 18: 2905-2912, 2012.

34. Amin S, Mhango G, Lin J, Aronson A, Wisnivesky J, Boffetta P and Lucas AL: Metformin improves survival in patients with pancreatic ductal adenocarcinoma and pre-existing diabetes: A propensity score analysis. Am J Gastroenterol 111: 1350-1357, 2016.

35. Chaiteerakij R, Petersen GM, Bamlet WR, Chaffee KG, Zhen DB, Burch PA, Leof ER, Roberts LR and Oberg AL: Metformin use and survival of patients with pancreatic cancer: A cautionary lesson. J Clin Oncol 34: 1898-1904, 2016.

36. Lee MS, Hsu CC, Wahlqvist ML, Tsai HN, Chang YH and Huang YC: Type 2 diabetes increases and metformin reduces total, colorectal, liver and pancreatic cancer incidences in Taiwanese: A representative population prospective cohort study of 800,000 individuals. BMC Cancer 11: 20, 2011.

37. Lu S and Archer MC: Sp1 coordinately regulates de novo lipogenesis and proliferation in cancer cells. Int J Cancer 126: 416-425, 2010.

38. Nair V, Pathi S, Jutooru I, Sreevalsan S, Basha R, Abdelrahim M, Samudio I and Safe S: Metformin inhibits pancreatic cancer cell and tumor growth and downregulates Sp transcription factors. Carcinogenesis 34: 2870-2879, 2013.

39. Zhang Y, Storr SJ, Johnson K, Green AR, Rakha EA, Ellis IO, Morgan DA and Martin SG: Involvement of metformin and AMPK in the radioresponse and prognosis of luminal versus basal-like breast cancer treated with radiotherapy. Oncotarget 5: 12936-12949, 2014.

40. Lonardo E, Cioffi M, Sancho P, Sanchez-Ripoll Y, Trabulo SM, Dorado J, Balic A, Hidalgo M and Heeschen C: Metformin targets the metabolic achilles heel of human pancreatic cancer stem cells. PLoS One 8: e76518, 2013.

41. Soares HP, Ni Y, Kisfalvi K, Sinnett-Smith J and Rozengurt E: Different patterns of Akt and ERK feedback activation in response to rapamycin, active-site mTOR inhibitors and metformin in pancreatic cancer cells. PLoS One 8: e57289, 2013

42. Dowling RJ,Zakikhani M, Fantus IG, Pollak M and Sonenberg N Metformin inhibits mammalian target of rapamycin-dependent translation initiation in breast cancer cells. Cancer Res 67: 10804-10812, 2007.

43. El-Mir MY, Nogueira V, Fontaine E, Avéret N, Rigoulet M and Leverve X: Dimethylbiguanide inhibits cell respiration via an indirect effect targeted on the respiratory chain complex I. J Biol Chem 275: 223-228, 2000. 